\title{
- The Five Ds for Rational use of Antimicrobial Agents
}

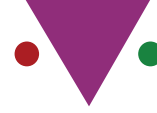

IJCRR

Section: Healthcare

ISI Impact Factor

(2020-21): 1.899

IC Value (2020): 91.47

SJIF (2020) $=7.893$

(c) (i) (9)

Copyright@IJCRR

\section{Pramod Kumar Manjhi}

Professor (Assistant) and Head, Department of Pharmacology, All India Institute of Medical Sciences, Patna-801507 (Bihar), India.

E-mail: drpramodkumar@aïmspatna.org

\section{INTRODUCTION}

In 1985 WHO, conference of experts at Nairobi defined the rational use of medicines as "patients receive medications appropriate to their clinical needs, in doses that meet their own individual requirements, for an adequate period of time, and at the lowest cost to them and their community. ${ }^{1}$ In simplest words rational use means prescribing right drug, inadequate dose for the sufficient duration and appropriate to the clinical needs of the patient at lowest cost. Inappropriate use of antimicrobials for non-bacterial infections, often in inadequate dose, non-adherence to dosing regimens, over-use of injections and non-compliance of prescription writing in accordance with clinical guidelines are considered irrational use of antimicrobial agents.

World Antimicrobial Awareness Week (WAAW) is celebrated from 18-24 November every year. The 2021 theme, Spread Awareness, Stop Resistance, calls on One Health stakeholders, policymakers, health care providers, and the general public to be Antimicrobial Resistance (AMR) Awareness champions. $^{2}$

Improving use of antibiotics through antibiotic stewardship is one of the key interventions necessary to curb the further emergence and spread of antimicrobial resistance (AMR). It is also important for ensuring appropriate treatment. For that reason, WHO in 2017 introduced the Access, Watch, Reserve ("AWaRe") classification of antibiotics in its Essential Medicines List. The classification is a tool for antibiotic stewardship at local, national and global levels with the aim of reducing antimicrobial resistance. ${ }^{3}$

Practically speaking there are 5Ds that can help clinicians to use antimicrobial therapy. They are: 1. Decision 2. Drug 3. Dosing 4. De- escalation 5. Duration

\section{HOW CAN HELP?}

I. Decision: It is most critical in the context of antimicrobial therapy. Selection of the most appropriate drug requires knowledge of 1) Patient factors like age, host defence status, renal \& hepatic dysfunction, allergy, pregnancy \& lactation, 2) Microbe factors which should be sensitive selected drugs based on culture \& sensitivity and minimum inhibitory concentration (MIC) \& minimum bactericidal concentration (MBC), 3) Drug factors including spectrum, dose \& duration. Ideally, the antimicrobial agent used to treat an infection is selected after the organism has been identified and its susceptibility to antimicrobial agents established. However, in the critically ill patient, such a delay could prove fatal, and immediate empiric therapy is indicated. Antibiotics used for empirical therapy are usually broad-spectrum antibiotics and should be substituted with narrow-spectrum antibiotics within 48 to 72 hours as per culture and sensitivity report. Because microbiological results do not become available for 24 to 72 hours, initial therapy for infection is often empiric and guided by the clinical presentation. It has been shown that inadequate therapy for infections in critically ill, hospitalized patients is associated with poor outcomes, including greater morbidity and mortality as well as increased length of stay.

II. Drug: Selection of right drug depends on appropriate diagnosis and therapeutic objectives whether definitive, empirical or prophylactic therapy. Choice of right molecule based on mechanism of action, susceptibility, side effects and pharmacokinetic parameters. Drug should be efficacious, tolerable, safe, suitable and cost effective for the particular patient. Selected drug should be specific having status of first line.

III. Dosing: Dosing of antimicrobial agents is based on pharmacodynamics (the relationship of drug concentrations to antimicrobial effects) and pharamacokinetic properties (the absorption, distribution, metabolism, and elimination of the drug). Three important properties that have a significant influence on the frequency of dosing are concentration-dependent killing (e.g. aminoglycosides \& quinolones), time-dependent killing (e.g. beta lactam antibiotics, macrolides, vancomycin), and postantibiotic effect (e.g. aminoglycosides \& quinolones). Utilizing these properties 
to optimize antibiotic dosing regimens can improve clinical outcomes and possibly decrease the development of resistance.

IV. De- escalation: De-escalate antimicrobials or step down to the narrowest spectrum, most efficacious, and most costeffective option as per culture reports. Parenteral therapy is normally used in seriously ill patients and those with gastrointestinal upset. Switch over from intravenous to oral therapy in case of oral route is not compromised (that is, no vomiting, nil by mouth, severe diarrhoea, swallowing disorders, unconscious)

V. Duration: The duration of therapy for many infections has long been based on anecdotal data and expert opinion. In view of the deleterious effects of prolonged courses of antimicrobial agents, including the potential for adverse reactions, problems with adherence, selection of antibioticresistant organisms, and high cost, a number of studies have tried to define the optimal duration of therapy, with an emphasis on shorter courses of therapy. For example, evidence supports limiting treatment of uncomplicated UTI in women to 3 days, community-acquired pneumonia to 5 days and ventilator-associated pneumonia to 8 days. $^{4}$

\section{CONCLUSION}

Rational use of antimicrobials is a holistic approach including clinical decision of right diagnosis, drug of choice, appropriate dose and duration of treatment as well as de-escalation ( 5Ds) as soon as appropriate. The final goal is to optimize therapy in order to maximize efficacy and minimize side effects and emergence of resistance.

\section{REFERENCES}

1. Rational use of medicines, available at https://www.who.int/activities/promoting-rational-use-of-medicines. [ Last accessed on 2022 Jan. 26]

2. World Antimicrobial Awareness Week (WAAW), available at https:/www.who.int/campaigns/world-antimicrobial-awareness-week/2021. [ Last accessed on 2022 Jan. 26]

3. The 2019 Who Aware Classification of Antibiotics for Evaluation and Monitoring of Use. World Health Organization; Geneva, Switzerland: 2019.

4. R. Leekha S, Terrell CL, Edson RS. General principles of antimicrobial therapy. Mayo Clin Proc. 2011;86(2):156-167. doi:10.4065/mcp.2010.0639 